\title{
APROPIACIÓN SOCIAL DE LAS NUEVAS TECNOLOGÍAS DE LA INFORMACIÓN Y LAS COMUNICACIONES (TICS) E INFORMATIZACIÓN DE LA SOCIEDAD COLOMBIANA'
}

\section{Fernando Chaparro Osorio}

\section{RESUMEN}

La llamada "Nueva Economía", que utiliza de manera amplia la información y el conocimiento como factores de producción, ha generado un rápido progreso científico y tecnológico, a la vez que un volumen sin precedentes de innovaciones industriales y transformaciones en las relaciones mundiales.

En este nuevo contexto, un elemento esencial de la competitividad y la sostenibilidad de las sociedades que están surgiendo es su capacidad de innovación, pues esta les permite traducir las oportunidades generadas por los desarrollos científicos y tecnológicos en nuevos productos y servicios, en posibilidades de trabajo y en un mayor bienestar para la sociedad.

El surgimiento de "nuevas tecnologías" hace parte del proceso. Ellas generan empresas, permean los sectores tradicionales de la producción, y ocasionan incluso, desempleo como parte de la reestructuración competitiva de los sectores económicos que las utilizan.

Existen tres grandes áreas tecnológicas: las tecnologías de la información y las comunicaciones (TICS), la biotecnología, producida por la de biología molecular, y los nuevos materiales. Estas áreas son intensamente científicas; no representan un sector económico, sino que se extienden a todos los sectores de la producción y de la sociedad; generan un alto valor agregado, tanto en las actividades productivas como sociales, y tienen gran influencia en la comunidad, en el mercado de trabajo, actividades culturales y formas de participación social.
El artículo trata en especial del caso de las TIC, aunque hay muchas sinergias que surgen de la interacción entre estos tres sectores, como es el caso de la bioinformática y de sus aplicaciones en el manejo de la biodiversidad. También detalla cómo enfrenta Colombia la "sociedad del conocimiento" y como se transforma paulatinamente al desarrollar su infraestructura tecnológica y de comunicación.

\section{PALABRAS CLAVE}

Nueva economía, sociedad del conocimiento, tecnologías de comunicación e información (TICs), innovación, conectividad, globalización, revolución digital.

\section{ABTSRACT}

The so called "New Economy", which extensively uses information and knowledge as production factors, has generated rapid scientific and technological progress, as well as an unprecedented volume of industrial innovations and transformations in worldwide relations.

In this new context, an essential element of competitiveness and sustainability of societies which are rising is their capacity for innovation, because this allows them to translate opportunities generated by scientific and technological developments into new products and services, into possibilities for work and greater welfare for society.

The rise of "new technologies" is part of the process. They generate companies, permeate traditional production sectors, and even cause unemployment as a part of the competitive restructuring of the economic sectors using them.

1. Conferencia realizada por Corporación Escenarios, Politécnico Grancolombiano y Portafolio. Bogotá, abril 1 de 2004 
Un elemento esencial de la competitividad y la

sostenibilidad delas sociedades es el desu capacidad de innovación que les permita traducir las oportunidades ocasionadas porlos desarrollos científicos $y$ tecnológicos en nuevos productosy servicios, yen posibilidades de trabajo y de bienestar para la sociedad.
There are three large technological areas: information and communications technologies (ICT), biotechnology, produced by molecular biology, and new materials. These are intensely scientific areas; they do not represent an economic sector, but rather, they extend to all sectors of production and society; they generate high added value, both in productive as well as social activities, and they have great influence on the community, the labor market, cultural activities and types of social participation.

\section{TICs, GLOBALIZACIÓN Y DESAFÍOS DEL NUEVO ENTORNO}

Los últimos 20 años se han caracterizado por una dramática evolución que nos ha llevado de una estructura productiva inten-

\section{RESEÑA AUTOR:}

Luis Fernando Chaparro Osorio, sociólogo de la Universidad de Lovaina, Bélgica, realizó su Máster en Sociología en la Universidad de Princeton, Estados Unidos, y fue becado por esa misma institución para cursar el Ph.D. en Sociología Industrial y Organizacional.

Fernando Chaparro dirige actualmente el Centro de Gestión del Conocimiento y la Innovación (CGCI) de la Universidad del Rosario. En su carrera profesional ha desempeñado importantes cargos directivos en diversas organizaciones, tales como Director para América Latina y el Caribe del Centro Internacional de Investigaciones para el Desarrollo (CIID/IDRC) del Canadá (1981-1992); Director General de la Corporación Colombiana de Investigaciones Agropecuarias - CORPOICA (1992-1994); Director General del Instituto Colombiano para el Desarrollo de la Ciencia y la Tecnología - COLCIENCIAS de 1994 a 1998; Secretario General del Foro Global de Investigaciones Agropecuarias (FGIA/GFAR), con sede en la FAO en Roma, Italia (1998 - 2002); Director de la Fundación Planeta Valle de Cali (2002-2003); y Director Ejecutivo de la Corporación Colombia Digital (2003-2004).

Fue miembro de la Misión de Educación, Ciencia y Desarrollo convocada por el Presidente de Colombia César Gaviria, Presidente de la Junta Directiva del Centro de Investigación sobre Agricultura Tropical (CIAT) y ha sido consultor internacional, conferencista y autor de numerosos artículos y ensayos sobre desarrollo científico y tecnológico, planeación y evaluación de la investigación, desarrollo organizacional de institutos tecnológicos y cooperación técnica internacional. Actualmente es miembro de numerosas asociaciones científicas y tecnológicas de Colombia y de América Latina.
The article especially deals in the case of the ITC's, although there are many synergies which come about from the interaction between these three sectors, as in the case of bioinformatics and its applications in the management of biodiversity. It also details how Colombia faces the "knowledge society" and how it is gradually transformed by developing its technology and communications infrastructure.

siva en capital y en uso de recursos energéticos, dominada por producción masiva en cadena, productos estandardizados, competencia estructurada y grandes organizaciones (empresas y agencias del Estado), hacia el surgimiento de la llamada "Nueva Economía” basada en el uso intensivo de información y conocimiento como factores de producción, y caracterizada por un rápido progreso científico y tecnológico que ha generado un volumen sin precedentes de innovaciones industriales y ha logrado transformar el contexto mundial en el que vivimos. Está emergiendo una economía fundamentada en la capacidad de generar y usar conocimiento, en la calidad de sus recursos humanos, en altos requerimientos de capacidad empresarial, la cual exige un marco institucional ágil y flexible que responda a los profundos cambios dados en el mercado de trabajo y en el entorno. En este nuevo contexto, un elemento esencial de la competitividad y la sostenibilidad de las sociedades es el de su capacidad de innovación que les permita traducir las oportunidades ocasionadas por los desarrollos científicos y tecnológicos en nuevos productos y servicios, y en posibilidades de trabajo y de bienestar para la sociedad. Esta última 
es un eslabón esencial para que los avances de la ciencia y la tecnología se traduzcan en desarrollo socio-económico efectivo.

Como parte de este proceso aparecen un conjunto de nuevas tecnologías que no solo generan empresas sino que crecientemente permean los sectores tradicionales de la producción, inclusive producen desempleo en ciertas ocasiones como parte de la reestructuración competitiva que dicho proceso desata. Nos referimos aquí a tres grandes sectores o áreas tecnológicas: las tecnologías de la información y las comunicaciones (TICs), la biotecnología causada por la revolución de la biología molecular y los nuevos materiales. Estos nuevos sectores tecnológicos tienen varias características en común: son científicamente intensivos; no representan un sector de la producción, sino que cortan a través de prácticamente todos los sectores de la producción y de la sociedad, permeando esta última; generan un alto valor agregado tanto en las actividades productivas como sociales; $y$ tienen un alto impacto en diversas esferas de la sociedad, incluso en el mercado de trabajo, en aspectos culturales y formas de participación social. En este artículo nos concentraremos en el caso de las TICs, aunque hay muchas sinergias que surgen de la interacción entre estos tres sectores, como es el caso de la bioinformática y de sus múltiples aplicaciones en el manejo de la biodiversidad.

Las tecnologías de la información y las comunicaciones tienen un profundo impacto en todos los sectores de la actividad humana, desde la producción hasta la educación y los servicios de salud. La convergencia de tres áreas tecnológicas anteriormente diferenciadas, que son la informática (computadores), las telecomunicaciones y la transferencia y procesamiento de datos y de imágenes, ha llevado a profundos cambios en la producción de bienes y servicios en las sociedades contemporáneas. La capacidad de acceso y de uso de dichas tecnologías, como son la microelectrónica, las diversas tecnologías relacionadas con la multimedia, y la multiplicidad de aplicaciones en la educación, la salud, la producción, los servicios y el control ambiental, dan lugar al proceso de informatización de la sociedad. La terciarización de la economía, inclusive en el sector agropecuario y manufacturero, es uno de los aspectos estrechamente vinculados con este proceso de informatización ${ }^{2}$.

La revolución causada por las tecnologías de la información y las comunicaciones ha procurado, por primera vez en la historia de la humanidad, la posibilidad de movernos en la dirección de una verdadera sociedad global, basada en la capacidad para interactuar en tiempo real a través de Internet o del correo electrónico entre personas y grupos geográficamente dispersos. La globalización es un proceso que va más allá de la apertura de mercados y de la internacionalización de la economía. Responde al proceso que Arnold Toynbee en su magna obra, A Study of History, planteó como uno de los principales desafíos de la humanidad al final del siglo XX: la construcción de una sociedad global, que requiere una institucionalidad y un marco normativo que complemente al estado-nación y los mecanismos intergubernamentales que han históricamente

2. Para un análisis del papel que las tecnologías de la información y las comunicaciones están desempeñando en la construcción de sociedades del conocimiento, con una capacidad de innovación, ver: Robin Mansell y Uta When (eds.). Knowledge Societies: Information Technology for Sustainable Development. London: Oxford University Press, 1998. 
La confluencia entre internety la multiplicidad de tecnologías y aplicaciones que están surgiendo transforman cualitativamente una parte muy importante de las actividades socioeconómicas en todas las sociedades que las adoptan. dominado el ámbito internacional. Se trata del surgimiento de la sociedad civil transnacional que se refleja en la globalización de los mercados, de la producción y de los sistemas financieros. Pero igualmente este proceso se refleja en la globalización de la ciencia, en la cual la comunidad científica desempeña un papel de creciente importancia.

Es importante resaltar el hecho de que la globalización no sólo toca a aquellos que participan en este nuevo entorno. Incide así mismo en los sectores sociales que no tienen la posibilidad de insertarse y participar activamente en ella, pero que sufren sus consecuencias a través de procesos que esta última genera, como es el caso del desempleo causado por la reestructuración competitiva, de cambios en los mercados de trabajo y en las competencias laborales que se requieren, $\mathrm{o}$ de procesos de transformación institucional de ella derivados. Los procesos de exclusión social que esto causa, pueden llegar a tener un impacto más significativo que los procesos de participación que dicho entorno facilita.

Para responder con éxito al desafío de desarrollar en Colombia una Sociedad del Conocimiento con capacidad para usar creativamente las nuevas tecnologías, surgen dos grandes dimensiones que inciden en este proceso. El desafío de la conectividad, medido por el grado de desarrollo de la infraestructura tecnológica y de comunicaciones requerida, el grado de penetración de internet y el desarrollo de un marco institucional y normativo que facilite el uso de esta tecnología por parte de empresas, gobierno, ONGs y comunidades urbanas y rurales. En segundo lugar, el desafío de la informatización real de la sociedad y de la economía, proceso mucho más complejo que requiere un proceso de apropiación social de estas nuevas tecnologías por los diversos actores sociales y sectores de la sociedad, llevando a una integración creativa e innovadora de dichas tecnologías en las formas de actuar de estos últimos. Lo cual implica un cambio cultural en organizaciones, en comunidades y en personas. $\mathrm{Al}$ respecto, Manuel Castells plantea muy claramente que:

La idea que la revolución tecnológicoinformativa, a partir de un determinado momento y con base en una cierta transformación organizativa de empresas y cultural de sociedades pueda empezar a proporcionar el dividendo de la productividad ya se puede observar... Lo anterior está abriendo la posibilidad de un salto histórico en la relación entre forma de generación económica y generación de riqueza (valor agregado, productividad y crecimiento) ${ }^{3}$.

Más recientemente este salto histórico del cual habla Manuel Castells ha sido analizado por Nicolás Curien y Pierre-Alain Muet, en un penetrante análisis que presentaron en $\mathrm{La}$ Société de l'Information, ${ }^{4}$ informe preparado para el Consejo de Análisis Económico (CAE) de Francia. La tesis principal de este informe es que la revolución digital no es meramente un cambio tecnológico importante en el sector de la informática y las comunicaciones, sino que se trata de la Tercera Revolución Industrial. La confluencia entre internet y la multiplicidad de tecnologías y aplicaciones que están surgiendo transforman cualitativamente una parte muy importante de las actividades socio-económicas en todas las sociedades que las adoptan. La gestión en

3. Manuel Castells. "La ciudad de la Nueva Economía”. En: La Factoría, No. 12, junio-septiembre del 2000.

4. Nicolás Curien y Pierre-Alain Muet. La Société de l'Information. Paris: Conseil d'Analyse Economique (CAE), 2004. 
red de empresas y de clusters regionales (networked firms), la integración de la robótica y otras TICs en la producción y los sistemas de inteligencia tecnológica y competitiva -gestión del conocimiento-, modifican el ámbito de la producción y del trabajo con profundos impactos en la productividad y en las competencias laborales requeridas. Con base en la evidencia empírica de países europeos, los autores plantean que el paradigma de Robert Solow ya no se aplica, aunque tuvo una validez histórica. Al final de los ochenta Solow había constatado que inversiones importantes que se estaban haciendo en computadores y en adopción de software no estaban generando un crecimiento de la productividad o de la competitividad en dichas ramas industriales como se había esperado. Es evidente que en ese momento se adoptaba muy primaria y simplemente las nuevas tecnologías, sin percatarse de que, para que ellas tuvieran efecto, era necesario vincularlas a estrategias integradas de innovación y productividad de las empresas, lo cual levaba muy a menudo a repensar el "business" mismo de la empresa -los cambios organizacionales y culturales a los que hace referencia Castells-. En su reciente estudio Nicolás Curien y PierreAlain Muet constatan que, cuando eso se da, se desencadena una dinámica diferente que se puede observar en el hecho de que en los últimos cinco años el $0.35 \%$ del crecimiento anual de Francia, Alemania e Italia se debe al creciente uso de estas tecnologías, y en el caso de Estados Unidos este impacto representa el $0.95 \%$ del crecimiento anual. Y que los sectores de la producción que no están adoptando este enfoque se están quedando por fuera de dicha dinámica. En el sector social el impacto es igualmente profundo, al generar nuevas formas de participación ciudadana y de comunidades de aprendizaje que pueden democratizar el conocimiento y empoderar comunidades con base en la utilización creativa de las nuevas tecnologías en la solución de problemas y en movilizar actores sociales alrededor de acciones concertadas. Tal es el tema de las TICs y de las colectividades o comunidades locales.

En el Gráfico No. 1 vemos una representación del proceso de "informatización de la sociedad" cortando a través de diversos sectores de una sociedad, aspecto que va mucho más allá del tema de conectividad. El impacto de estas tecnologías se siente no sólo en la producción y en gobierno-en-línea, dimensiones en las cuáles estas tecnologías hacen un aporte significativo, sino que igualmente tienen un impacto importante en las otras esferas de la sociedad: la cultura, la salud, la educación, el manejo sostenible del medio ambiente y la propia dinámica social (por ejemplo, participación social y movimientos sociales), que pueden potencializarse con un uso creativo de las nuevas tecnologías al fortalecer su capacidad de acceso a y gestión del conocimiento, así como de creación del mismo a través de procesos de aprendizaje social.

\section{Gráfico No. 1-Diversos Aspectos en la Informatización de la Sociedad}

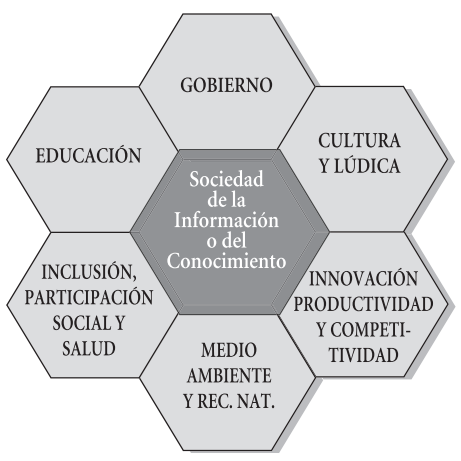


Pero si bien estas tecnologías abren grandes oportunidades, igualmente generan desafíos y peligros que pueden llevar a consecuencias muy diferentes según el contexto en el que se dan, y según la capacidad de los actores sociales para apropiarse de ellas e integrarlas con sus propios objetivos. Aquí surge una serie de preguntas básicas:

¿Cómo hacer paraque las nuevas tecnologías puedan potencializar y resaltar la cultura propia, $y$ se conviertan en un instrumento de expresión de ella y de protección de la diversidad cultural, $y$ no en un instrumento de homogeneización $y$ de alienación? a. ¿Cómo lograr ese proceso de apropiación social real de la tecnología para que pueda surtir sus efectos positivos? b. ¿Cómo evitar las fuerzas o tendencias de "exclusión social" que a menudo diferencian a los que dominan y saben cómo aprovechar dichas tecnologías de los que quedan excluidos (la "brecha digital")? El desafío de la inclusión social y el aporte que las TICs pueden hacer a dicho objetivo, es uno de los principales retos que actualmente confrontamos. c. ¿Cómo integrar el uso creativo de las TICs en estrategias integradas de innovación y productividad, no sólo en las empresas sino en cadenas de producción y de clusters regionales (networked firm)? ¿Y cómo hacerlo para que el pequeño productor no quede excluido de este proceso, agravado el creciente problema de pobreza que confrontan nuestras sociedades? Es decir, este enfoque es tan relevante para las grandes cadenas agroindustriales de naturaleza transnacional como para las minicadenas locales: el comercio electrónico y los mercados digitales pueden ser tan útiles para los unos como para los otros. d. ¿Cómo podemos transformar la escuela sobre la base del uso innovador de las TICs para que esta desarrolle los recursos humanos y las personas creativas con capacidad de aprender-a-aprender y de manejar conocimiento, requerido por el nuevo entorno? e. ¿Cómo hacer para que las nuevas tecnologías puedan potencializar y resaltar la cultura propia, y se conviertan en un instrumento de expresión de ella y de protección de la diversidad cultural, y no en un instrumento de homogeneización y de alienación? f. ¿Cómo aprovechar las nuevas tecnologías para crear nuevas empresas y oportunidades de trabajo, pese al desempleo que la inevitable reestructuración competitiva puede generar? ¿Cuáles son las competencias laborales que este entorno crea y cómo podemos responder a los profundos cambios en el mercado de trabajo? g. En fin, ¿cómo podemos aprovechar estas tecnologías para construir los nuevos instrumentos de la globalización y de la sociedad global, que respondan a los requerimientos de las sociedades locales, a su diversidad cultural, a nuevas formas de valorar el conocimiento local tradicional, al mismo tiempo que lo integramos y lo complementamos con el conocimiento científico universal?

En su clásica historia de la tecnología, el profesor Paul E. Landes de la Universidad de Harvard destacó que no hay tecnologías neutras en la historia de la humanidad que generen resultados automáticos y asegurados, ni que puedan ser consideradas como panaceas para los múltiples problemas que se confrontan en cualquier época histórica. Las grandes revoluciones tecnológicas abren oportunidades y ciclos que las sociedades deben reconocer y aprovechar, pero depende de ellas que utilicen creativamente estos paradigmas emergentes en la construcción de su futuro ${ }^{5}$. Las innovaciones sociales o la reorganización social que las nuevas tecnologías generan son tan importantes o más importantes que los propios cambios tecnológicos. El desafío que confrontamos es el de construir una Sociedad del Conocimiento que sea equitativa y soste-

5. Paul E. Landes. The Unbound Prometheus. Boston: Harvard University Press, 1968. 
nible, basada en los principios del desarrollo humano y de la inclusión social, en el contexto actual de la globalización y de mercados altamente competitivos.

\section{GRADO DEINFORMATIZACIÓN DELA SOCIEDAD COLOMBIANA}

En este contexto surge la pregunta sobre el grado de preparación de la sociedad colombiana para responder con éxito a las oportunidades y desafíos que el nuevo entorno engendra. La respuesta es diferente para las dos grandes dimensiones identificadas en la sección anterior: el tema de la conectividady el desafío de la informatización y la apropiación social de las nuevas tecnologías.

En lo referente al tema de conectividad, Colombia ha hecho avances significativos en los últimos años gracias a la labor desarrollada por la Agenda de Conectividad, el Ministerio de Comunicaciones por parte del gobierno, y por las empresas de telecomunicaciones a través de sus programas de masificación de Internet por parte del sector productivo (Empresa de Teléfono de Bogotá, Empresas Públicas de Medellín, Empresa Regional de Telecomunicaciones del Valle del Cauca, etc.). A pesar de este esfuerzo, Colombia sigue rezagada en relación con otros países de similar nivel de desarrollo. Hay dos tipos de indicadores que se pueden utilizar para medir el grado de conectividad de una sociedad. En primer lugar, hay un conjunto de indicadores simples que miden el grado de penetración o adopción de ciertas tecnologías por parte de la sociedad. Los cuatro indicadores estandar que se utilizan son los del número de computadores, número de líneas telefónicas fijas, número de teléfonos celulares y número de usuarios de internet por cada 1.000 habitantes. Estos indicadores simples tienen claras limitaciones por el hecho de que sólo toman en consideración la dimensión puramente tecnológica y no reflejan la complejidad y las diversas dimensiones de la sociedad de la información o del conocimiento, pero tienen la gran ventaja de que son fáciles de medir y se pueden utilizar para hacer comparaciones en el tiempo o en el espacio (entre países o regiones). En el Cuadro No 1 se resumen estos cuatro indicadores simples para ocho países de América Latina y el Caribe, presentándolos en orden descendente.

Cuadro $N^{\circ} 1$ Indicadores Simples de Conectividad

\begin{tabular}{|l|c|c|c|c|}
\hline \multicolumn{1}{|c|}{ País: } & $\begin{array}{c}\text { No. Compu- } \\
\text { tadores } \mathbf{x} \\
\mathbf{1 , 0 0 0 :}\end{array}$ & $\begin{array}{c}\text { No. Teléfonos } \\
\text { fijos x 1,000: }\end{array}$ & $\begin{array}{c}\text { No. Teléfonos } \\
\text { celulares } \mathbf{x} \\
\mathbf{1 , 0 0 0 :}\end{array}$ & $\begin{array}{c}\text { No. Usuarios } \\
\text { de Internet } \mathbf{x} \\
\mathbf{1 , 0 0 0 :}\end{array}$ \\
\hline Chile & 119 & 230 & 428 & 237.5 \\
\hline Costa Rica & 197 & 251 & 111 & 193.1 \\
\hline México & 82 & 147 & 255 & 98.5 \\
\hline Uruguay & 110 & 280 & 193 & 119 \\
\hline Brasil & 74.8 & 223 & 201 & 82.2 \\
\hline Argentina & 82 & 219 & 178 & 112 \\
\hline Colombia & 49.3 & 179 & 106 & 46.2 \\
\hline Perú & 43 & 66 & 86 & 93.5 \\
\hline Am. Latina (prom.) & 69 & 166 & 191 & 81.2 \\
\hline
\end{tabular}

Fuente: PNUD: Informe sobre Desarrollo Humano 2004, New York, 2004.

(Ver: http://hdr.undp.org/reports/global/2004).

United Nations Statistics Division, New York, 2004. (Ver: http://unstats.un.org/unsd) 
Cuadro No. 2 Indicadores Simples de Conectividad por Región del Mundo

Es interesante destacar el caso de Costa Rica, pues siendo éste un país pequeñole ha dado una alta prioridad a utilizar las nuevas tecnologías como motor de desarrollo, y ha combinado éste enfoque con una estrategia de desarrollo igualmente basada en recursos naturales que le asigna un importante papel al sector agropecuario.

\begin{tabular}{|l|c|c|c|c|}
\hline $\begin{array}{l}\text { Región o Grupo } \\
\text { de Países: }\end{array}$ & $\begin{array}{c}\text { No. } \\
\text { Compu- } \\
\text { tadores } \mathbf{x} \\
\mathbf{1 , 0 0 0 :}\end{array}$ & $\begin{array}{c}\text { No. } \\
\text { Teléfonos } \\
\text { fijos } \mathbf{x} \\
\mathbf{1 , 0 0 0 :}\end{array}$ & $\begin{array}{c}\text { No. Teléfonos } \\
\text { celulares } \mathbf{x} \\
\mathbf{1 , 0 0 0 :}\end{array}$ & $\begin{array}{c}\text { No. } \\
\text { Usuarios de } \\
\text { Internet } \mathbf{x} \\
\mathbf{1 , 0 0 0 :}\end{array}$ \\
\hline $\begin{array}{l}\text { Regiones alto nivel } \\
\text { de desarrollo }\end{array}$ & 364 & 507 & 582 & 382.6 \\
\hline $\begin{array}{l}\text { América Latina y el } \\
\text { Caribe }\end{array}$ & 69 & 166 & 191 & 81.2 \\
\hline $\begin{array}{l}\text { Europa Central y } \\
\text { Oriental \& CEl }\end{array}$ & --- & 226 & 189 & 71.8 \\
\hline $\begin{array}{l}\text { Asia Oriental y el } \\
\text { Pacífico }\end{array}$ & 26 & 142 & 159 & 60.9 \\
\hline Estados Árabes & --- & 81 & 85 & 28.0 \\
\hline Asia Meridional & 10 & 41 & 13 & 14.9 \\
\hline $\begin{array}{l}\text { África } \\
\text { Subsahariana }\end{array}$ & 12 & 15 & 39 & 9.6 \\
\hline Prom. Mundial & 10 & 175 & 184 & 99.4 \\
\hline
\end{tabular}

Fuente: United Nations Statistics Division, New York, 2004.

(Ver: http://unstats.un.org/unsd)

Como se puede observar en el Cuadro No 1, Colombia está significativamente por debajo de un buen número de países de América Latina, como es el caso de Chile, Costa Rica, México, Uruguay, Brasil y Argentina. Dicho atraso es especialmente notorio en el número de usuarios de Internet: 46.2 por 1.000 habitantes en comparación con 98.5 en México, 193.1 en Costa Rica y 237.5 en Chile. El Cuadro No 2 suministra las cifras relevantes para los países desarrollados. Aun la cifra más reciente del 2004 de 69 usuarios por 1.000 sigue siendo baja. En los otros indicadores se observa un atraso similar. Es interesante destacar el caso de Costa Rica, pues siendo éste un país pequeño le ha dado una alta prioridad a utilizar las nuevas tecnologías como motor de desarrollo, y ha combinado éste enfoque con una estrategia de desarrollo igualmente basada en recursos naturales que le asigna un importante papel al sector agropecuario. Es de señalar que las nuevas tecnologías se pueden combinar con una estrategia de desarrollo fundamentada en recursos naturales y en el papel estratégico que pueden desempeñar ciertas agroindustrias, como igualmente lo demuestra el caso de Chile. Se deben evitar "opciones falsas" que buscan anteponer estrategias de desarrollo cimentadas en las nuevas tecnologías a otras basadas en sectores tradicionales y en recursos naturales. Como se indicó en la primera sección, una característica esencial de las nuevas tecnologías es que cortan a través de todos los sectores de la economía, incluyendo los tradicionales, transformándolos y dinamizándolos. Lo que sí se requiere es integrar explícitamente las nuevas tecnologías a las estrategias de desarrollo, cualesquiera que sea la base económica de estas últimas. 
El contexto colombiano está rápidamente cambiando como lo muestran las cifras más recientes del Ministerio de Comunicaciones. En el caso del número de teléfonos celulares por 1.000 habitantes, Colombia pasó de 100 en diciembre del 2002 a 170 en julio del 2004. El número de usuarios de internet pasó de 1.6 millones en junio del 2002 a 3.1 millones en diciembre del 2003, lo que representa un crecimiento del 96\% en 18 meses (y llegó a 69 usuarios por 1.000 habitantes). En el Cuadro No 1 hemos dejado las cifras de Naciones Unidas, ya que en este mismo período se puede haber dado un incremento similar en los otros países. Por lo tanto, un cambio de las cifras en uno sólo de ellos puede desvirtuar la posibilidad de análisis comparativo, pero sí debe resaltarse el hecho de que la situación en Colombia está cambiando muy rápidamente. En el Cuadro No 2 se puede ver cómo se compara América Latina con las otras regiones del mundo en estos cuatro indicadores.

Además de los indicadores simples, se han realizado diversos esfuerzos para desarrollar indicadores compuestos - multivariados- que buscan reflejar la mayor complejidad de las TICs y las sociedades del conocimiento. El trabajo más sistemático que se ha hecho para desarrollar un índice de esta naturaleza es el llamado Network Readiness Index (NRI) desarrollado por la Universidad de Harvard y el Banco Mundial, cuyos resultados se publican anualmente en el Global Information Technology Report, publicación conjunta del Banco Mundial y del Foro Económico Mundial con el objetivo de suministrar información comparativa sobre el grado de adopción y uso de estas tecnologías en todos los países, tanto desarrollados como en vía de desarrollo. La principal característica de este índice es que no hace énfasis exclusivo en la tecnología y en su grado de uso, sino en las capacidades por desarrollar y en el entorno favorable que esto requiere. Por lo tanto, hace énfasis en los requerimientos institucionales y culturales que los cambios tecnológicos crean.

Es también interesante resaltar la variable o capacidad crítica que este indicador destaca: la capacidad de interactuar en red (network readiness), como aspecto esencial del nuevo entorno. No se trata meramente de tener computadores y saberlos utilizar; se trata de una actitud basada en una visión del mundo y de su entorno, en donde la cooperación y la interacción entre empresas o entre organizaciones académicas, y su inserción en redes, se considera el aspecto más importante de la nueva sociedad. Un interesante informe de la Unión Europea sobre la Sociedad del Conocimiento resalta la misma dimensión:

En las economías contemporáneas... el conocimiento crecientemente se codifica y se transmite a través de redes formales e informales que integran empresas, proveedores, distribuidores, centros tecnológicos y la propia comunidad. En este contexto la innovación se dinamiza a partir de la interacción entre generadores y usuarios del conocimiento, intercambiando entre sí tanto conocimiento codificado como conocimiento personal; este modelo interactivo ha remplazado el tradicional modelo lineal de innovación ${ }^{6}$.

6. Unión Europea. The Learning Society. Bruselas: Unión Europea, 1998. 
En la sección 3.1 se analizarán algunos de los proyectos innovadores por medio de los cuales se está buscando desarrollar en Colombia un uso innovador de las TICs en el sector productivo, vinculando esto último a una estrategia integrada de fomento a la innovación y la productividad.

El Network Readiness Index (NRI) se mide por medio de 48 variables organizadas alrededor de tres dimensiones básicas: a. Indicadores de entorno: entorno de mercado, entorno normativo y de política, y entorno de infraestructura existente. b. Indicadores de Capacidades Requeridas: capacidades individuales (educación), capacidades empresariales y capacidades del gobierno. c. Indicadores de Uso de las Tecnologías: uso individual, uso corporativo (empresarial) y uso por parte del gobierno. El Informe Anual del Banco Mundial -Global Information Technology Report-suministra información sobre este índice para 102 países del mundo, y los ubica en un ranking según la posición que cada país ocupa con relación a cada variable. Así mismo, suministra indicadores compuestos que presentan una síntesis al nivel de cada una de las tres dimensiones y al nivel global (el NRI). Cada indicador se expresa en términos de su valor numérico y de su ranking relativo en el conjunto de los 102 países.

En el Cuadro No 3 se presenta la información para Colombia con relación a las 48 variables que integra este indicador. En este cuadro se pueden ver cuáles son las variables que el Banco Mundial y el Foro Económico Mundial utilizan para "medir" cada dimensión. Por ejemplo, la calidad del "Entorno de Mercado se mide a través de nueve variables que incluyen no sólo aspectos relacionados con el mercado de productos derivados de las TICs (por ejemplo, exportación de productos/servicios TICs), sino también con la calidad del entorno científico-tecnológico que existe en dicho sector, medido a través de: calidad de las instituciones de IyD en el sector, número de científicos e ingenieros, número de patentes otorgadas, existencia de subsidios para IyD en la empresa, disponibilidad de capital de riesgo y otros factores similares. Es muy importante resaltar que estos son los factores que se consideran como críticos o estratégicos para poder desarrollar una capacidad de proyección con éxito en el nuevo entorno. De igual forma se puede ver en este cuadro cuáles son las variables a través de las cuáles se mide la calidad de los otros entornos.

Para cada una de estas variables, el Cuadro No 3 suministra información sobre el ranking relativo de Colombia en el contexto de los países que se incluyen en este análisis anual, e identifica en cuál aspectos somos menos "competitivos". Es así como se puede observar que, entre los 102 países, Colombia está bastante bien en lo referente a la "Presencia del Gobierno en-línea" (posición 24), aunque en lo referente a los "Servicios del Gobierno efectivamente enlinea" el posicionamiento es mucho menos favorable (74). La penetración de la radio es altísima y coloca al país en la posición 37, mientras que la penetración domestica de Internet es especialmente baja, y nos ubica en la posición 91 entre 102; es éste el aspecto en el que más atraso tenemos en comparación con los otros países. Igualmente baja es nuestra capacidad de exportaciones per cápita en el sector de las TICs, que nos 
mantiene en la posición 86. Colombia está mucho mejor, en términos relativos, en lo referente al marco jurídico de las TICs (posición 41) y en el grado de competencia que se ha logrado desarrollar entre los ISP (posición 45). Por lo tanto, detrás del indicador agregado (el NRI) es posible hacer un análisis más pormenorizado de las fortalezas y debilidades del país tal como esto se refleja en su posicionamiento entre los 102 países analizados por el Banco Mundial. La base de datos que esta agencia internacional ha construido sobre dichos aspectos suministra información más desagregada de cómo se midieron tales variables.

En el Cuadro No 4 se presenta la información para 29 de los 102 países que nos permite comparar a Colombia con un grupo representativo de países agrupados en tres grandes categorías: el grupo de los seis países más desarrollados en este campo; el conjunto de países que están ubicados entre los rangos 10 al 40; y el conjunto de países que se ubican por encima de la posición 40 (de 40 a 102). Dicho cuadro presenta tanto el valor numérico de los respectivos indicadores como la posición relativa de cada país en términos del ranking. En este cuadro podemos ver que Colombia ocupa la casilla 60 en el ranking del índice total (NRI), ocupa la posición 73 en los Indicadores de Uso Efectivo de la Tecnología, y la 45 en los Indicadores de Capacidades Requeridas de (Readiness Indicators). Aquí surge un contraste muy interesante. El mayor atraso del país se encuentra en el proceso de apropiación social de la tecnología y en su integración efectiva en el sector productivo y en los demás sectores de la vida nacional (posición 73); en contraste con esto, Colombia tiene una importante capacidad de uso de dichas tecnologías en términos de la calidad de sus recursos humanos y de su desarrollo institucional (se ubica en la posición 45 en éste último) (ver Cuadro No 4). En la sección 3.2 analizaremos algunos de los proyectos más innovadores en Colombia en lo referente a integrar el uso creativo de las TICs en fortalecer mecanismos de participación social en comunidades urbanas y rurales, fortaleciendo de esta forma el capital social de dichas comunidades.

Como se puede ver, estos indicadores sirven no sólo para fines del análisis comparativo entre países y para determinar la ubicación de Colombia en el ámbito mundial, sino también para identificar "factores de éxito" y "limitantes" que pueden facilitar u obstaculizar el desarrollo del país en este campo. Por lo tanto, este conjunto de indicadores/variables es de gran utilidad para establecer metas y derroteros para las políticas que el país debe desarrollar en este campo, así como para poder medir el grado de avance que logremos a través de dichas políticas.

\section{ALGUNOS EJEMPLOS DEAPROPIACIÓN SOCIAL DELAS TICS EN COLOMBIA}

En la primera sección y en el Gráfico 1 se destacó la forma como las tecnologías de la información y las comunicaciones influyen la sociedad en su conjunto e inciden en las diversas dimensiones de la sociedad, incluyendo al sector productivo, el suministro de servicios por parte del gobierno, la cultura, la salud, la educación, el manejo sostenible del medio ambiente y la propia dinámica social (por ejemplo, participación social y movimientos sociales). Las formas tradicionales de participación social pueden potencializarse con un uso creativo de las
El mayor atraso del país se encuentra en el proceso de apropiación social de la tecnología y en su integración efectiva en el sector productivo $y$ en los demás sectores de la vida nacional 
Las formas tradicionales de participación social pueden potencializarse

con un uso creativo de las nuevas tecnologías por el papel que estas últimas pueden desempeñar en facilitar nuevos espacios de diálogo entre ciudadanosy entre actores sociales, así como nuevos mecanismos de concertación que pueden fortalecer redes ciudadanas y generar procesos de aprendizaje social. nuevas tecnologías por el papel que estas últimas pueden desempeñar en facilitar nuevos espacios de diálogo entre ciudadanos y entre actores sociales, así como nuevos mecanismos de concertación que pueden fortalecer redes ciudadanas y generar procesos de aprendizaje social. En esta sección analizaremos dos casos innovadores que están surgiendo en Colombia: el primero se refiere a un proyecto orientado a desarrollar una plataforma electrónica que permita el desarrollo de mercados digitales estrechamente relacionados con clusters regionales en ciertos sectores de la producción; el segundo se refiere al uso innovador de las TICs en el desarrollo de creatividad en la comunidad, en el apoyo a comunidades virtuales de aprendizaje y en el fortalecimiento de redes ciudadanas tanto en comunidades urbanas como rurales, con el fin de fortalecer el capital social de estas últimas. Mientras el primer caso está relacionado con el tema de productividad y competitividad, el segundo tiene que ver con el aporte de las TICs al desarrollo de sociedades más democráticas y participativas, basadas en los principios del desarrollo humano y de la equidad.
3.1. TICS PRODUCTIVIDAD, INNOVACLÓN Y COMPEIIIIVIDAD

Hay dos grandes dimensiones que aparecen en la interacción entre las tecnologías de la información y las comunicaciones y el sector productivo. El primero es el surgimiento de nuevos sectores de la producción, como es el caso de las industrias del software, de la electrónica, de las comunicaciones y de un conjunto de servicios conexos que se han desarrollado como consecuencia directa de los nuevos cambios tecnológicos y de los productos y servicios de ellos derivados. Una creciente proporción de la producción mundial está relacionada con estos sectores tecnológicos y un número importante de países están desarrollando con éxito estrategias de industrialización y de inserción en los mercados globalizados del siglo XXI basadas en estas nuevas ramas de la producción. Es el caso de países como Irlanda, la India, Malasia, Singapur y otros, cuyas estrategias de desarrollo se centran en estos nuevos productos y servicios. Esta primera dimensión ciertamente desempeña un papel de gran importancia en las economías del conocimiento. 
Cuadro No. 3 Los Diversos Indicadores del NRI - Colombia, 2003

\begin{tabular}{|c|c|c|c|c|c|}
\hline I. Indicadores del Entorno & 64 & $\begin{array}{c}\text { II. Indicadores de } \\
\text { Capacidades Requeridas }\end{array}$ & 45 & $\begin{array}{l}\text { III. Indicadores de Uso de la } \\
\text { Tecnología }\end{array}$ & 73 \\
\hline 1. Entorno del Mercado: & 68 & $\begin{array}{l}\text { 4. Capacidades } \\
\text { individuales: }\end{array}$ & 52 & 7. Uso Individual: & 64 \\
\hline $\begin{array}{l}\text { 1.1 Estado del Desarrollo del } \\
\text { Cluster }\end{array}$ & 58 & $\begin{array}{l}\text { 4.1 Gasto Público en } \\
\text { Educación (per capita), } 2000\end{array}$ & 60 & $\begin{array}{l}\text { 7.1 Computadores Personales } \\
\text { (x } 1.000 \text { hab.), } 2001\end{array}$ & 55 \\
\hline $\begin{array}{l}\text { 1.2 Disponibilidad de Capital de } \\
\text { Riesgo }\end{array}$ & 69 & $\begin{array}{l}4.2 \text { Indice de } \\
\text { analfabetismo(\%), } 2001\end{array}$ & 55 & $\begin{array}{l}\text { 7.2 Suscriptores a ISDN } \\
(\mathrm{x} 1.000 \text { hab.), } 2001\end{array}$ & 41 \\
\hline $\begin{array}{l}\text { 1.3 Subsidios para Investigación y } \\
\text { Desarrollo a Nivel Empresarial }\end{array}$ & 71 & $\begin{array}{l}\text { 4.3 Vinculación al Tercer } \\
\text { Sector (\% Bruto), } 2001\end{array}$ & 55 & $\begin{array}{l}\text { 7.3 Suscriptores a Televisión } \\
\text { por Cable (x1.000 hab.), } 2001\end{array}$ & 71 \\
\hline $\begin{array}{l}\text { 1.4 Calidad de las Instituciones } \\
\text { de Investigación Científica }\end{array}$ & 64 & $\begin{array}{l}4.4 \text { Radios (x } 1.000 \text { hab.), } \\
2001 \text { o el más reciente }\end{array}$ & 37 & $\begin{array}{l}\text { 7.4 Usuarios de Internet (x } \\
1.000 \text { hab.), } 2001\end{array}$ & 62 \\
\hline $\begin{array}{l}\text { 1.5 Número de Científicos e } \\
\text { Ingenieros (x 1,000 hab.). }\end{array}$ & 74 & $\begin{array}{l}\text { 4.5 Televisores (x1.000 } \\
\text { hab.),2001 }\end{array}$ & 51 & 8. Uso Empresarial: & 71 \\
\hline 1.6 Fuga de Cerebros & 58 & $\begin{array}{l}\text { 4.6 Hogares en Línea (\% de } \\
\text { hogares con computadores), } \\
2002\end{array}$ & 91 & $\begin{array}{l}\text { 8.1 Computadores para } \\
\text { Negocios (x 1.000 hab.), } 2002\end{array}$ & 53 \\
\hline $\begin{array}{l}\text { 1.7 Número de Patentes } \\
\text { Concedidas } \\
\text { (x } 1.000 .000 \text { hab.), } 2002\end{array}$ & 59 & $\begin{array}{l}\text { 4.7 Calidad de la Educación } \\
\text { en Matemáticas y Ciencias, } \\
2003\end{array}$ & 58 & $\begin{array}{l}\text { 8.2 Absorción de la Tecnología } \\
\text { a Nivel Empresarial, } 2003\end{array}$ & 59 \\
\hline $\begin{array}{l}\text { 1.8 Exportación de Manufacturas } \\
\text { de Tecnologías de la Información } \\
\text { y Comunicación (per capita), } \\
2001\end{array}$ & 63 & $\begin{array}{l}\text { 4.8 Acceso a Línea } \\
\text { Telefónicas Reparadas (\% } \\
\text { per capita GDP), } 2001\end{array}$ & 46 & $\begin{array}{l}\text { 8.3 Prevalencia de } \\
\text { Licenciamiento de Tecnología } \\
\text { Extranjera, } 2003\end{array}$ & 75 \\
\hline $\begin{array}{l}\text { 1.9 Exportación de Servicios de } \\
\text { Tecnologías de la Información } \\
\text { y la Comunicación (per } \\
\text { capita), } 2001\end{array}$ & 86 & $\begin{array}{l}4.9 \text { Acceso Telefónico a } \\
\text { Internet(\% per capita GDP), } \\
2001\end{array}$ & 31 & 9. Uso Gubernamental: & 68 \\
\hline $\begin{array}{l}\text { 2. Entorno Normativo y de } \\
\text { Política: }\end{array}$ & 60 & $\begin{array}{l}\text { 4.10 Acceso a los precios de } \\
\text { los Proveedores de Servicios } \\
\text { de Internet } \\
\text { (\% per capita GDP), } 2001\end{array}$ & 69 & $\begin{array}{l}\text { 9.1 Éxito Gubernamental en } \\
\text { la Promoción de Tecnologías } \\
\text { de la Información y } \\
\text { Comunicación, } 2003\end{array}$ & 56 \\
\hline $\begin{array}{l}\text { 2.1 Total de Carga } \\
\text { Administrativa, } 2003\end{array}$ & 64 & & & $\begin{array}{l}\text { 9.2 Servicios del Gobierno } \\
\text { efectivamente en Línea, } 2003\end{array}$ & 74 \\
\hline $\begin{array}{l}\text { 2.2 Calidad en el Sistema Legal, } \\
2003\end{array}$ & 70 & 5. Capacidad Empresarial & 52 & & \\
\hline $\begin{array}{l}\text { 2.3 Leyes Relacionadas con las } \\
\text { Normas de las Tecnologías de la } \\
\text { Información y la Comunicación, } \\
2003\end{array}$ & 41 & $\begin{array}{l}\text { 5.1 Facilidad para la } \\
\text { Obtención de Líneas } \\
\text { telefónicas, } 2003\end{array}$ & 48 & & \\
\hline
\end{tabular}




\begin{tabular}{|c|c|c|c|}
\hline $\begin{array}{l}\text { 2.4 Grado de Competencia en el } \\
\text { Sector de ISP, } 2003\end{array}$ & 45 & $\begin{array}{l}\text { 5.2 Costo de la Suscripción } \\
\text { Mensual de Líneas de } \\
\text { Teléfonos Comerciales (\% } \\
\text { per capita GDP), } 2002\end{array}$ & 48 \\
\hline $\begin{array}{l}2.5 \text { Restricciones para } \\
\text { Inversionistas Extranjeros, } 2003\end{array}$ & 79 & $\begin{array}{l}\text { 5.3 Alcance del } \\
\text { Entrenamiento del } \\
\text { Empleado, } 2003\end{array}$ & 52 \\
\hline $\begin{array}{l}\text { 2.6 Eficiencia del Sistema de } \\
\text { Recaudación de Impuestos, } 2003\end{array}$ & 72 & $\begin{array}{l}\text { 5.4 Calidad en las Escuelas } \\
\text { de Negocios, } 2003\end{array}$ & 41 \\
\hline 2.7 Libertad de Prensa, 2003 & 49 & $\begin{array}{l}\text { 5.5 Científicos e Ingenieros } \\
\text { en Investigación y } \\
\text { Desarrollo (x } 1.000 \text { hab.), } \\
2000\end{array}$ & 86 \\
\hline 3. Entorno de Infraestructura & 64 & & \\
\hline $\begin{array}{l}\text { 3.1 Total de la Calidad de la } \\
\text { Infraestructura, } 2003\end{array}$ & 66 & 6. Capacidades del Gobierno & 39 \\
\hline $\begin{array}{l}\text { 3.2 Tiempo de Espera } \\
\text { para obtención de Líneas } \\
\text { Telefónicas(años), } 2000\end{array}$ & 71 & $\begin{array}{l}\text { 6.1 Priorización del } \\
\text { Gobierno en las Tecnologías } \\
\text { de la Información y la } \\
\text { Comunicación, } 2003\end{array}$ & 57 \\
\hline $\begin{array}{l}\text { 3.3 Líneas Telefónicas Principales } \\
\text { (x1.000 hab.), } 2001\end{array}$ & 55 & $\begin{array}{l}\text { 6.2 Presencia del Gobierno } \\
\text { en Línea, } 2003\end{array}$ & 24 \\
\hline $\begin{array}{l}\text { 3.4 Teléfonos Públicos (x } 1.000 \\
\text { hab.), } 2001\end{array}$ & 72 & $\begin{array}{l}\text { 6.3 Disponibilidad } \\
\text { Gubernamental de } \\
\text { Productos de Tecnología } \\
\text { de Información y } \\
\text { Comunicación, } 2003\end{array}$ & 64 \\
\hline $\begin{array}{l}\text { 3.5 Servidores de Internet (x } \\
1.000 \text { hab.), } 2001\end{array}$ & 59 & & \\
\hline
\end{tabular}

Fuente: Banco Mundial y Foro Económico Mundial:

The Global Information Technology Report 2003-2004; Washington, 2004. 
Cuadro No. 4 El Network Readiness Index (NRI) - 2003

\begin{tabular}{|c|c|c|c|c|c|c|c|c|}
\hline & \multicolumn{2}{|c|}{$\begin{array}{c}\text { Indicadores de } \\
\text { Entorno }\end{array}$} & \multicolumn{2}{|c|}{$\begin{array}{c}\text { Indicadores de } \\
\text { Capacidad }\end{array}$} & \multicolumn{2}{|c|}{ Indicadores de Uso } & \multicolumn{2}{|c|}{ NRI Total } \\
\hline & Score & Ranking & Score & Ranking & Score & Ranking & Score & Ranking \\
\hline $\begin{array}{l}\text { Estados } \\
\text { Unidos }\end{array}$ & 5.17 & 1 & 5.95 & 3 & 5.39 & 1 & 5.50 & 1 \\
\hline Singapur & 5.12 & 2 & 5.85 & 4 & 5.21 & 2 & 5.40 & 2 \\
\hline Finlandia & 4.98 & 3 & 6.07 & 1 & 4.63 & 9 & 5.23 & 3 \\
\hline Suecia & 4.72 & 6 & 5.95 & 2 & 4.94 & 5 & 5.20 & 4 \\
\hline Dinamarca & 4.61 & 10 & 5.81 & 5 & 5.15 & 3 & 5.19 & 5 \\
\hline Canadá & 4.67 & 7 & 5.66 & 8 & 4.88 & 6 & 5.07 & 6 \\
\hline Islandia & 4.84 & 5 & 5.28 & 16 & 4.52 & 14 & 4.88 & 10 \\
\hline Alemania & 4.42 & 17 & 5.5 & 12 & 4.62 & 10 & 4.85 & 11 \\
\hline Japón & 4.34 & 19 & 5.51 & 11 & 4.56 & 11 & 4.8 & 12 \\
\hline Taiwán & 4.66 & 8 & 5.25 & 17 & 3.95 & 22 & 4.62 & 17 \\
\hline Francia & 4.27 & 23 & 5.66 & 7 & 3.87 & 25 & 4.6 & 19 \\
\hline Irlanda & 4.28 & 22 & 5.24 & 18 & 4.13 & 18 & 4.55 & 22 \\
\hline Estonia & 4 & 25 & 5.11 & 22 & 3.65 & 27 & 4.25 & 25 \\
\hline Malasia & 3.95 & 26 & 4.86 & 29 & 3.78 & 26 & 4.19 & 26 \\
\hline España & 3.86 & 30 & 5.00 & 24 & 3.17 & 32 & 4.01 & 29 \\
\hline Italia & 3.89 & 28 & 4.91 & 26 & 3.41 & 29 & 4.07 & 28 \\
\hline Chile & 3.85 & 31 & 4.73 & 30 & 3.24 & 31 & 3.94 & 32 \\
\hline Sur África & 3.68 & 33 & 4.33 & 46 & 3.15 & 33 & 3.72 & 37 \\
\hline Brasil & 3.66 & 35 & 4.49 & 40 & 2.85 & 47 & 3.67 & 39 \\
\hline México & 3.36 & 47 & 4.29 & 47 & 3.05 & 36 & 3.57 & 44 \\
\hline India & 3.45 & 44 & 4.23 & 50 & 2.94 & 44 & 3.54 & 45 \\
\hline Costa Rica & 3.37 & 46 & 4.14 & 55 & 2.87 & 46 & 3.46 & 49 \\
\hline Argentina & 3.15 & 57 & 4.24 & 49 & 2.97 & 42 & 3.45 & 50 \\
\hline China & 3.03 & 63 & 4.14 & 54 & 2.97 & 43 & 3.38 & 51 \\
\hline Uruguay & 3.25 & 52 & 4.18 & 53 & 2.63 & 61 & 3.35 & 54 \\
\hline Colombia & 3.02 & 64 & 4.34 & 45 & 2.48 & 73 & 3.28 & 60 \\
\hline Egipto & 3.08 & 60 & 3.86 & 71 & 2.62 & 63 & 3.19 & 65 \\
\hline Perú & 2.83 & 71 & 3.97 & 66 & 2.48 & 72 & 3.09 & 70 \\
\hline Ecuador & 2.57 & 90 & 3.19 & 93 & 2.27 & 83 & 2.68 & 89 \\
\hline
\end{tabular}

Fuente: Banco Mundial y Foro Económico Mundial: The Global Information Technology Report 2003-2004; Washington, 2004.

En abril del 2004 la Corporación Colombia Digital (CCD), el SENA y Colciencias organizaron una serie de talleres de trabajo para analizar la experiencia de Irlanda en la formulación de una
Estrategia Nacional Regional orientada al desarrollo de una economía del conocimiento, basada en las Nuevas Tecnologías, y de los instrumentos y mecanismos a través de los cuales ella se materializa. Para tal fin 
se invitó al doctor David O'Donovan, que fue uno de los principales arquitectos de esta experiencia nacional en su calidad de director del Investment Development Agency (IDA) de Irlanda. En los talleres de trabajo se analizó dicha experiencia, sobretodo en lo referente a la creación de nuevas empresas, tanto empresas nacionales como multinacionales establecidas en Irlanda a través del fomento a la inversión extranjera (FDI) y de mecanismos de cofinanciación (matching grants) y de capital de riesgo nacional. La estrategia que Irlanda ha seguido en las tres últimas décadas se ha concentrado básicamente en los sectores dinámicos de la Nueva Economía (las llamadas Nuevas Tecnologias): software e industria electrónica (TICs), biotecnología e industria farmaceútica. Dicha estrategia se orientó a la construcción de una capacidad nacional que le permitió a Irlanda competir con éxito en el nuevo contexto de la "economía del conocimiento", por medio de una fuerte inversión en educación, en capacidades endógenas en las nuevas áreas de la ciencia y la tecnología, y en una política muy articulada que vinculó este esfuerzo con la creación de nuevas empresas en estos sectores. No se trata solamente del desarrollo de una capacidad científica y tecnológica en estos campos, sino en su sistemática vinculación con la producción y la generación de empleo, y por lo tanto, con la innovación. Esto último, complementado con una clara focalización en áreas prioritarias (propósito nacional y regional), logró movilizar un consenso social, y le permitió a la estrategia irlandesa un efecto importante. Los resultados son claros al pasar de una economía tradicional a una economía moderna en tres décadas; logró un ingreso per cápita de US $\$ 40.000$ y un superavit en la balanza comercial de 46.5 billones de dólares, con una población de menos de 4 millones de personas. Para mayor información sobre esta experiencia, incluyendo diagnósticos y documentos de política de éste país, ver la página web de la CCD, especialmente www.colombiadigital.net/futuroes/experiencia_irlanda.php .

Sin embargo, hay una segunda dimensión que es aún más importante que la primera, como claramente lo demuestra el reciente informe de Nicolás Curien y Pierre-Alain Muet preparado para el Consejo Económico de Francia citado en la nota al pie de página 3. Se trata del impacto de las nuevas tecnologías en todos los sectores de la producción, incluyendo los tradicionales, tales como confecciones, textiles, turismo, agroindustrias de diversos tipos, manejo de recursos naturales, etc. Es aquí donde estamos viendo el mayor impacto de transformación que están teniendo las nuevas tecnologías, al redefinir la forma como las empresas interactúan con su entorno, con sus clientes y por lo tanto, con su propio negocio. Por eso en la sección 2 se destacó el hecho de que hay países como Costa Rica y Chile que han combinando el desarrollo de las nuevas tecnologías con una estrategia de desarrollo igualmente basada en recursos naturales, en la que se le asigna un papel importante al sector agropecuario. Por lo tanto se deben evitar alternativas o dicotomías falsas que buscan anteponer estrategias de desarrollo basadas en las nuevas tecnologías a otras basadas en sectores tradicionales y en recursos naturales, como si fueran excluyentes. Una característica esencial de las nuevas tecnologías es que cortan a través de todos los sectores 
de la economía, incluyendo los tradicionales, transformándolos y dinamizándolos. Lo que sí se requiere es integrar explícitamente las nuevas tecnologías a las estrategias de desarrollo, cualesquiera sea la base económica de estas últimas.

Por esta razón la Corporación Colombia Digital (CCD) y otras entidades plantean la necesidad de vincular el desarrollo de las TICs a un enfoque integrado de fomento a la innovación y a la productividad. Cuando se analiza el uso de las TICs en el sector productivo en forma aislada (por ejemplo, grado de uso de computadores, adquisición de software empresarial, uso de internet, etc.), se pierde de vista la contribución que estas tecnologías dinamizan y fortalecen la competitividad. Nos referimos aquí al papel de las TICs en el "nuevo ámbito de la innovación", en el que el manejo del conocimiento en la empresa y en cadenas de producción está desempeñando un papel de creciente importancia. Las múltiples aplicaciones de las tecnologías de la información y las comunicaciones (TICs) en el sector productivo incluyen aspectos tales como servicios de información, inteligencia de mercados, sistemas de información geo-referencial, automatización industrial, sistemas de control, uso de estas tecnologías en el control de calidad, nuevas formas de participación en los procesos de toma de decisiones, nuevas estructuras empresariales y de mercadeo que dichas tecnologías hacen posible, etc. Esta gran diversidad de aplicaciones están íntimamente vinculadas a procesos de innovación que están transformando el sector productivo en la industria, en el sector agropecuario, en el sector de transportes y en el sector energético, constituyéndose en factor esencial de productivi- dad y competitividad en dichos sectores de la producción.

Por lo tanto, para comprender el papel real de estas tecnologías y aprovecharnos de su potencialidad, es necesario analizarlas en el contexto de un enfoque integrado de fomento a la innovación y la productividad de las empresas, usando las TICs como un instrumento para lograr este objetivo. Es interesante resaltar el hecho de que varios estudios recientes sobre empresas innovadoras en los países de la OECD han demostrado claramente que la innovación ya no se relaciona solamente con innovación de producto e innovación de proceso, sino que el comportamiento innovador de la empresa crecientemente se basa en su capacidad para manejar información y conocimiento sobre mercados, sobre nuevas oportunidades y sobre cambios en su entorno, convirtiendo la capacidad de "gestión del conocimiento" de la empresa o de una cadena de producción -gestión en red- en un factor crítico de productividad y competitividad. Esta visión es mucho más compleja y rica que la de simplemente facilitar el acceso a internet por parte de empresas, o el de adoptar software empresarial en estas últimas (por ejemplo, CRM, ERP).

Por tal razón, es muy importante complementar los programas que Confecámaras, con el apoyo de la Agenda de Conectividad, está desarrollando a través de proyectos como el de Prymeros, con un enfoque más integral por medio de proyectos experimentales en algunas regiones del país, tales como el de la Plataforma Tecnológica e Informática de Apoyo al Cluster Regional de Desarrollo Agroindustrial de Bogotá y Cundinamarca. Éste último forma parte de un proyecto más grande que se denomina Megaproyecto
Varios estudios recientes sobre empresas innovadoras en los países de la OECD han demostrado claramente que la innovación ya no se relaciona solamente con innovación de producto e innovación deproceso, sino que el comportamiento innovador de la empresa crecientemente se basa en su capacidad para manejar información y conocimiento sobre mercados, sobre nuevas oportunidades $y$ sobre cambios en su entorno, convirtiendo la capacidad de "gestión del conocimiento" de la empresa o de una cadena deproducción -gestión en red-en un factor crítico de productividady competitividad. 
de Desarrollo Agroindustrial de Bogotá y Cundinamarca, siendo uno de tres componentes de dicho proyecto ${ }^{7}$. Al cual lo impulsa la Cámara de Comercio de Bogotá y el CARCE de Bogotá, Cundinamarca, en colaboración con un grupo de empresarios. El establecimiento de esta plataforma electrónica e informática tiene tres objetivos: a. crear un espacio de Mercado Digital (Open Digital Marketplace) en el cual los empresarios, proveedores y distribuidores puedan hacer transacciones en tiempo real; b. facilitar el acceso a información pertinente y a servicios tecnológicos relacionados con la gestión del conocimiento; $y$ c. fortalecer la competitividad sistémica del cluster regional y su internacionalización a través del uso creativo de las TICs. Para lograr estos tres objetivos se están diseñando los siguientes componentes y servicios, como parte de esta plataforma ${ }^{8}$ :

- Desarrollar un Portal Interactivo y Transaccional (www.redilog.net) que ayude a articular una Comunidad de Productores y Comercializadores de Productos Agroindustriales de Bogotá y Cundinamarca. El portal existente es puramente informativo y no tiene la capacidad que está actualmente en construcción de "portal interactivo y transaccional".

- Desarrollar un Programa Central (software o "motor electrónico") lo suficientemente robusto como para facilitar el acceso a las bases de datos y a fuentes de información, y permitir la organización de Mercados Digitales a través de Internet. Aquí se buscará Integrar tecnología que se pueda traer de centros de excelencia mundial, como es el caso del Media Lab de MIT que tiene desarrollos interesantes en este campo, con las tecnologías y los productos que puede desarrollar la industria nacional de software y de electrónica. A través de esta Plataforma Tecnológica Informática se facilitará acceso a las empresas del cluster a servicios tecnológicos (ver siguientes puntos), utilizando internet como un medio.

- Integrar las diversas bases de datos existentes en un Sistema de Información Regional que incluya información sobre mercados, sobre zonas de producción por vocación agrícola, estadísticas de producción, producción exportable, oferta tecnológica (paquetes tecnológicos probados), información sobre el entorno y cambios en el entorno, etc. Además de integrar las existentes (por ejemplo, Proexport), se identificarán requerimientos de bases de datos o servicios de información que deban ser desarrollados o fortalecidos. Una de las bases de datos importantes será la de oferta tecnológica disponible en los cultivos relevantes, con base en los servicios de Corpoica, la SAG, en el Valle del Cauca, la CCI y otros similares.

- Fortalecer la capacidad de gestión de cultivos de cadenas de producción a través de instrumentos basados en las TICs, como Rediagro, que ya se están utilizando en algunos cultivos en el

7. Los otros dos componentes de este proyecto son: un Centro de Servicios Logísticos y un Centro de Apoyo Económico y Financiero.

8. Para mayor información sobre este proyecto ver: Desarrollo de una Plataforma Tecnológica e Informática de Apoyo al Cluster Regional de Desarrollo Agroindustrial de Bogotá y Cundinamarca. Bogotá: CCD, septiembre, 2003. 
país. Este programa ha sido desarrollado por el grupo Carulla y se está utilizando con éxito en diversos cultivos (como la cadena de la piña en el Valle del Cauca, etc.).

- Establecer un Servicio de Benchmarking (identificación de "buenas prácticas" y de "casos exitosos" de uso de las TICs en las empresas) e identificación de cuellos de botella o de limitantes tecnológicos en empresas, o de cluster regional agroindustrial. Esto se buscará integrar con el Sistema de Inteligencia Competitiva que la Cámara de Comercio de Bogotá está desarrollando con Qubit Cluster para la cadena hortifrutícola, el cual cubre tres aspectos: a. información económica, b. inteligencia de mercados y c. inteligencia tecnológica.

- Desarrollar/Fortalecer las capacidades de las empresas para usar las nuevas tecnologías y hacer una gestión eficiente de la información y el conocimiento (desarrollo de una nueva cultura empresarial). Esto implica poner enpráctica un programa de sensibilización sobre la importancia de estos servicios e instrumentos, así como capacitar recursos humanos en diferentes niveles y sobre diversos aspectos ( comercio electrónico, mercados digitales, gestión del conocimiento en la empresa, etc.). Se buscará desarrollar este componenete en cooperación con el SENA.

Hay otros proyectos similares que se están proponiendo en Antioquia, el Valle del Cauca, Caldas y el Caribe. Tales experiencias pueden fortalecerse con los enfo- ques innovadores desarrollados en diversas regiones de España, como es el caso del Politécnico de Barcelona y la Universidad de Valencia, donde se ha hecho gran énfasis en el tema de inteligencia tecnológica e inteligencia competitiva ${ }^{9}$, los cuales pueden aportar al desarrollo e implementación de la propuesta que actualmente se está discutiendo en Colciencias de desarrollar "Instrumentos para la Globalización", como parte de la política de innovación.

\section{TICS, DESARROLLO DE CAPITAL SOCIAL Y EMPODERAMIENTO DE LA COMUNIDAD}

El segundo caso innovador que analizaremos es el del uso de las tecnologías de la información y las comunicaciones (TICs) en el desarrollo de creatividad en la comunidad, en el apoyo a "comunidades virtuales de aprendizaje" y en el fortalecimiento de redes ciudadanas tanto en comunidades urbanas como rurales, con el fin de fortalecer el capital social de estas últimas. Uno de los impactos más importantes que las nuevas tecnologías están generando es el de "empoderar comunidades" por la capacidad que dichas tecnologías tienen para facilitar el proceso de "construcción de comunidades virtuales de aprendizaje" que pueden desempeñar tres funciones importantes: a. desarrollar una capacidad en la comunidad de generar y usar conocimiento útil para la solución de los problemas que confronta, utilizando enfoques construccionistas para diseñar tecnologías autóctonas que respondan a sus necesidades y requerimientos, combinando conocimiento "científico" con conocimiento tradicional; b. crear espacios de participación social y de diálogo entre

9. Pere Escorsa y Ramón Maspons. De la Vigilancia Tecnológica a la Inteligencia Competitiva. Madrid: Financial Times/Prentice-Hall, 2001. 
ciudadanos utilizando las nuevas tecnologías, facilitando la consolidación de foros de participación ciudadana y el acceso a información sobre buenas prácticas en términos de participación social, convivencia, gobernabilidad y construcción de capital social; y c. lograr una educación liberadora que pueda desarrollar creatividad y una capacidad de aprender a aprender en los niños y en los futuros ciudadanos, por medio del uso de las TICs para transformar la escuela desde adentro ${ }^{10}$. Por limitaciones de espacio, en este artículo analizaremos los dos primeros puntos relacionados con el uso de las TICs en la comunidad, no tratando el tema de la transformación de la escuela.

La Corporación Colombia Digital (CCD) está colaborando con otras entidades interesadas en la amplia apropiación social de las nuevas tecnologías con el fin de fomentar su uso en el desarrollo de una sociedad más participativa y democrática, como es el caso de la Universidad Autónoma de Occidente (UAO), Manizales Eje del Conocimiento (MEC), Infimanizales, el Ministerio de Educación, la Unesco, el Banco Mundial y otras. En el contexto de esta iniciativa se ha formulado una propuesta para desarrollar en Colombia un programa sobre: TICs, Construcción de Capital Social y Empoderamiento de la Comunidad. El objetivo de este programa es el de desarrollar un esfuerzo concertado orientado a adelantar en Colombia un proceso de apropiación social de las nuevas tecnologías en comunidades y grupos sociales marginados o vulnerables, que permita el uso creativo de estas tecnologías para desarrollar una sociedad más participativa y equitativa por medio de nuevas formas de participación social, de la creación de espacios de diálogo y de concertación ciudadana y del fortalecimiento de la capacidad de comunidades para generar y usar conocimiento que les sea útil en la solución de sus problemas y en incrementar su calidad de vida y su bienestar ${ }^{11}$. Este programa está constituido por una serie de proyectos específicos que se están desarrollando o se desarrollarán en diversas comunidades urbanas y rurales colombianas. En algunos casos se trata de proyectos que ya están en ejecución y que se están integrando a la iniciativa para que los unos puedan aprovechar las experiencias de los otros y buscar complementariedad entre ellos. En otros casos se trata de nuevos proyectos que se están proponiendo. Estos proyectos son:

- Establecimiento de un portal de la Comuna 13 de Medellín como plataforma para construir y consolidar espacios de participación ciudadana.

- Proyecto sobre el uso creativo de las TICs (video: Fortalecer Identidad Cultural en la Comuna 13 de Medellín).

- Proyecto: Construcción y Consolidación de Redes Ciudadanas Virtuales en el Distrito de Aguablanca de la Ciudad de Cali.

- Proyecto: Educación para la Paz: Establecimiento de un Centro de

10. Este es el principal tema del proyecto que la CCD está desarrollando con el Media Lab de MIT en Manizales en lo referente a la transformación de la escuela. Sobre éste proyecto ver Claudia Urrea. Improving Schools from the Inside-Out: The Role of Teacher and Digital Technologies. Cambridge: MIT, October, 2003.

11. Programa de Educación para la Paz: Tecnologías de la Información y las Comunicaciones (TICs), Construcción de Capital Social y Empoderamiento de la Comunidad. Bogotá: CCD, marzo del 2004. Para mayor información sobre este programa y los proyectos que lo constituyen ver la página web de la CCD, especialmente los proyectos descritos en: www.colombiadigital.net/global/noticias.php?nid=12 . 
Creatividad en la Comuna 5 de Manizales para empoderar la comunidad con uso de TICs (en cooperación con el Media Lab de MIT).

- Proyecto: Comunicación para la Vida orientado al desarrollo de una red de comunicación regional para las comunidades indígenas de Cauca, Valle y Chocó.

- Plan de Formación en Comunicación Indígena Waunaan del Bajo San Juan.

En el desarrollo de este programa dos grupos han desarrollado un papel de liderazgo: el Programa Comunicación para el Desarrollo de la Universidad Autónoma de Occidente (UAO) de Cali y Manizales Eje del Conocimiento (MEC), este último, con el apoyo de Infimanizales. En el caso de Medellín los proyectos han sido formulados por ONG locales bajo la coordinación de Realizadores de Sueños y de la CCD.

A partir de este esfuerzo se está desarrollando una red nacional entre los proyectos actualmente en ejecución, que permita compartir las experiencias, resultados y metodologías surgidas de proyectos innovadores que comparten el objetivo general de impulsar el uso creativo de las TICs para desarrollar capital social y empoderar la comunidad. Estos diversos proyectos y experiencias están combinando un conjunto de elementos claves que son los siguientes:

a) El establecimiento o fortalecimiento de un Centro Comunitario que facilite y promueva el acceso y uso creativo de las nuevas tecnologías por parte de los ciudadanos o pobladores de esa comunidad. En los diversos proyectos que están en proceso de gestación se han planteado "tipos diferentes" de centros comunitarios. Un tipo de centro comunitario es el que se está planteando para la Comuna 5 de Manizales, un Centro Comunitario de Creatividad (en cooperación con el MIT), centrado en el uso de la robótica para desarrollar soluciones autóctonas a los problemas que la comunidad confronta (de producción, de manejo sostenible de recursos naturales, etc.), relacionando estas tecnologías con procesos didácticos comunitarios basados en la teoría del construccionismo y orientados a desarrollar creatividad y una capacidad de uso innovador de estas tecnologías entre los muchachos y demás ciudadanos de la comunidad. En otros casos, el tipo de centro comunitario establecido es más cercano al tipo de Telecentros surgidos en forma experimental en los últimos dos años. La principal diferencia entre los dos es que los Telecentros no integran el componente de los instrumentos de la electrónica y microelectrónica ni los aspectos didácticos o pedagógicos orientados al desarrollo de la creatividad y al uso innovador de las nuevas tecnologías para "generar soluciones autóctonas locales”. Pero las demás funciones y servicios son similares. El CIAT y la Universidad Autónoma de Occidente (UAO) han desarrollado algunas de las experiencias más innovadoras con el apoyo del IDRC del Canadá, trabajando con comunidades urbanas y rurales del Cauca, Valle del Cauca y Chocó.

b) Las comunidades participantes han planteado la necesidad de establecer el Portal de la Comunidad como una pla- 
taforma electrónica que suministre un espacio virtual que sirva como un mecanismo de apoyo a las redes sociales de la comunidad y como un punto de convergencia para las organizaciones, habitantes, grupos culturales y procesos socioculturales de la comunidad. Proceso generalmente liderado por ONG de la

Un cuarto aspecto que está despertando mucho interés en las comunidades en las que se desarrolla el programa propuesto es el de establecer un periódico electrónico virtual operado por los propios ciudadanos, que suministre información oportunay veraz sobre los diferentes acontecimientos sociales, culturales, politicosy económicos de la comunidad. propia comunidad quien es la que está participando en el diseño de tales portales y en la definición de los contenidos que se pondrán en los mismos. A partir de este portal comunitario se facilitará, así mismo, acceso a otros portales nacionales que son de interés para los actores sociales de la comunidad (como el Portal Avanza que Colnodo promueve en apoyo a las ONG, el Portal Educativo Colombia Aprende del Ministerio de Educación, los observatorios ambientales apoyados por el Ministerio del Medio Ambiente y portales relacionados con cadenas de producción y temas de empleo que pueden ser de interés para muchas personas). Estos portales pueden facilitar acceso a fuentes de información del mundo globalizado relacionados con procesos similares que se están adaptando en otros países latinoamericanos o en otras regiones del mundo. Bien diseñado, el portal se puede convertir en un medio para dinamizar redes sociales en la comunidad. Una de las plataformas más ágiles que tenemos para este fin es Edunet, desarrollada por la ERT y la Universidad Autónoma de Occidente (UAO) con el apoyo de Colciencias. c) Con base en los dos primeros elementos, complementados con el uso de tecnologías de comunicación inalámbrica (WIFI), se busca apoyar el desarrollo y consolidación de "comunidades de aprendizaje" sobre temas de interés para cada comunidad y de redes ciudadanas que faciliten el surgimiento de foros de participación ciudadana. El objetivo que se persigue aquí es el de desarrollar procesos de aprendizaje en la comunidad relacionados con sus necesidades básicas, para fortalecer de esta manera la capacidad local de generar y utilizar conocimiento pertinente que le permita a la comunidad solucionar sus problemas y construir su futuro, evitando la tendencia a la exclusión social que genera la brecha digital. En estos casos se están aplicando las tecnologías desarrolladas por el Media Lab del MIT no solo en lo referente a comunicación inalámbrica (WIFI), sino también en lo referente a la llamada "Teoría de Comunicación Viral" como nuevo patrón de comunicación social generado por estas últimas.12 Los casos concretos que están surgiendo en las diversas comunidades se relacionan con temas de producción (por ejemplo, apoyar grupos de microempresarios buscando consolidar cadenas de producción locales), con temas de participación ciudadana a través de foros y redes que puedan fortalecerse con el uso creativo de las TICs, o con temas de desarrollo cultural, por medio del uso de video y otros medios de comunicación como mecanismo de

12. Andrew Lippman. Communications Futures. Cambridge: MIT, abril 2003. David P. Reed. Constructing a 'Communications Ether' that can Grow and Adapt. Cambridge: MIT, abril 2003. También se puede consultar en http://dl.media.mit.edu/viral. 
información y de desarrollo de identidad cultural. En este último tema una ONG de la Comuna 13 de Medellín está proponiendo un proyecto muy innovador de uso de video y otras tecnologías de comunicación, que incluye las tradicionales (radio), para desarrollar un programa de información sobre temas de la comunidad, de promoción e impulso de valores de convivencia y solidaridad, relacionados igualmente con temas de derechos humanos.

d) Un cuarto aspecto que está despertando mucho interés en las comunidades en las que se desarrolla el programa propuesto es el de establecer un periódico electrónico virtual operado por los propios ciudadanos, que suministre información oportuna y veraz sobre los diferentes acontecimientos sociales, culturales, políticos y económicos de la comunidad de interés para sus habitantes. Este periódico podría funcionar con una red de corresponsales que integre estudiantes de las escuelas o ciudadanos en general, y viabilice una red de medios. Ya hay propuestas concretas en los proyectos de Aguablanca en Cali, de la Comuna 13 de Medellín, en la Comuna 5 de Manizales y de Pensilvania (Caldas).

Estos cuatro elementos son los que se están utilizando en forma modular para usar creativamente las TICs en el apoyo a redes ciudadanas y a foros de partici- pación social que fortalezcan el capital social de las comunidades urbanas y rurales donde se aplican, así como el acceso a conocimiento útil para la comunidad a través de Internet o de mecanismos para sistematizar conocimiento local ${ }^{13}$. Este programa se presentó en el Séptimo Encuentro Iberoamericano del Tercer Sector que tuvo lugar en Sao Paulo, Brasil ( mayo 16-19 del 2004), y se destacó como uno de las propuestas más innovadoras presentados en dicho encuentro. Allí mismo se resaltó la importancia de crear una Red Iberoamericana que facilite la difusión de estas experiencias y la identificación de las "mejores prácticas", que genere una especie de benchmarking en este campo. Entre los países andinos, la Comunidad Andina de Naciones (CAN) y el Instituto de Conectividad de las Américas (ICA) están apoyando una iniciativa similar; iniciativas que pueden constituir redes regionales o globales sobre estos temas, contribuyendo a difundir las experiencias exitosas y a desarrollar sinergismos entre los diversos grupos y comunidades que están trabajando con procesos de apropiación social de estas tecnologías. Dichas redes también pueden jugar un papel importante en integrar experiencias aisladas en conocimiento codificado y transmisible, y generar procesos de aprendizaje social sobre el uso de las TICs en la construcción de capital social y en el empoderamiento de comunidades urbanas y rurales por medio del uso creativo de las nuevas tecnologías.

13. Sobre este último (sistematización del conocimiento local), existen enfoques innovadores y software especializado como el desarrollado por GRET, una ONG de Francia, a través de proyectos en diversos países en desarrollo. Esta experiencia se implementó en el contexto del Foro Global de Investigación Agropecuaria (GFAR). 
En el Séptimo Encuentro Iberoamericano del Tercer Sector, al que se ha hecho referencia, el principal factor que se resaltó fue el de la potencialidad de las nuevas tecnologías para desarrollar nuevas formas de participación ciudadana, y por lo tanto definir el nuevo tipo de ciudadano (o las nuevas formas de ciudadanía) que están surgiendo en las sociedades del conocimiento ${ }^{14}$. Este aspecto también surge con suma claridad en el conjunto de estudios recientemente realizados por el Consejo de Análisis Económico (CAE) de Francia sobre la Sociedad del Conocimiento en el contexto de las sociedades Europeas ${ }^{15}$.

14. Fernando Chaparro. Conocimiento y Tecnología: Ciudadanía, Desarrollo y Conectividad. Sao Paulo: Séptimo Encuentro Iberoamericano del Tercer Sector; Sao Paulo, mayo 16-19 del 2004 (se puede copiar de: www. colombiadigital.net/global/noticias.php?nid=9).

15. E. Éveno y D. Latouche. Les Collectivités Locales: Acteurs du Modèle Français de la Société de l'Information. Paris: Conseil d'Analyse Èconomique (CAE), 2004. Se puede copiar de la página web de la CCD: www. colombiadigital.net/docs/Collectivites.pdf. 